\title{
Early interruption of exclusive breastfeeding: results from the eight-country MAL-ED study
}

\author{
Crystal L Patil ${ }^{1}$, Ali Turab², Ramya Ambikapathi ${ }^{3}$, Cebisa Nesamvuni ${ }^{4}$, Ram Krishna Chandyo ${ }^{5}$, Anuradha Bose ${ }^{6}$, \\ M Munirul Islam ${ }^{7}$ AM Shamsir Ahmed ${ }^{8}$, Maribel Paredes Olortegui ${ }^{9}$, Milena Lima de Moraes ${ }^{10}$, Laura E Caulfield ${ }^{10^{*}}$, \\ on behalf of the MAL-ED network
}

\begin{abstract}
We report the infant feeding experiences in the first month of life for 2,053 infants participating in "Malnutrition and Enteric Infections: Consequences for Child Health and Development" (MAL-ED). Eight sites (in Bangladesh, India, Nepal, Pakistan, Brazil, Peru, South Africa, Tanzania), each followed a cohort of children from birth (by day 17), collecting detailed information on infant feeding practices, diet and illness episodes. Mothers were queried twice weekly regarding health status, breastfeeding and the introduction (or no) of non-breast milk liquids and foods. Here, our goal is to describe the early infant feeding practices in the cohort and evaluate factors associated with termination of exclusive breastfeeding in the first month of life. With data from enrollment to a visit at 28-33 days of life, we characterized exclusive, predominant or partial breastfeeding (using a median of 6-9 visits per child across the sites). Only 6 of 2,053 infants were never breastfed. By one month, the prevalences of exclusive breastfeeding were $<60 \%$ in 6 of 8 sites, and of partial breastfeeding (or no) were $>20 \%$ in 6 of 8 sites. Logistic regression revealed that prelacteal feeding (given to 4-63\% of infants) increased the likelihood of partial breastfeeding (Odds Ratio (OR): 1.48 (95\% confidence interval (Cl): 1.04, 2.10), as did the withholding of colostrum (2-16\% of infants) (OR: 1.63:1.01, 2.62), and being a first-time mother (OR: 1.38:1.10, 1.75). Our results reveal diversity across these sites, but an overall trend of early transition away from exclusive breastfeeding in the first month of life. Interventions which introduce or reinforce the WHO/UNICEF Ten Steps for Successful Breastfeeding are needed in these sites to improve breastfeeding initiation, to reinforce exclusive breastfeeding and delay introduction of non-breast milk foods and/or liquids.
\end{abstract}

Keywords: Breastfeeding, Prelacteal feeding, Colostrum, MAL-ED, Nepal, Bangladesh, Pakistan, India, Brazil, Peru, Tanzania, South Africa

\section{Introduction}

Overwhelming clinical and epidemiological evidence supports international recommendations for initiation of breastfeeding shortly after birth, avoidance of prelacteal feeding, and exclusive breastfeeding for six months, followed by the timely introduction of safe and nutritionally adequate complementary foods to prevent malnutrition and reduce morbidity in the first five years of life [1-4]. Optimal breastfeeding and the timely introduction of appropriate complementary foods not only decreases risk of diseases, especially from gastrointestinal and respiratory infections, but also promotes health and

\footnotetext{
* Correspondence: Icaulfie@jhsph.edu

${ }^{10}$ The Johns Hopkins Bloomberg School of Public Health, Department of International Health, 615 North Wolfe Street, W2041, Baltimore, MD, USA21205 Full list of author information is available at the end of the article
}

growth and development [5]. Of the 8-10 million of child deaths that occur annually, more than $90 \%$ of these deaths take place in developing countries [1]. Many of these deaths are linked to malnutrition and, in fact, an estimated $13 \%$ are related to suboptimal infant feeding practices [6,7]. Given this evidence, it is not surprising that globally, less than $25 \%$ of children 6 months of age are exclusively breastfed and for those 4 months of age, the percentage (35\%) is only slightly better [6].

The importance of breastfeeding initiation is highlighted in epidemiological studies from Ghana, India and Nepal which show a relationship between timing of initiation and risk for neonatal mortality [8-10]. In Ghana, low-risk infants were at twice the risk of neonatal death if they were not breastfed within the first 24 hours after birth. Even after controlling for potential confounding factors, 
late initiation was associated with a 78\% increased risk for mortality in India [11]. Although the causes of breastfeeding initiation delay were not explored, other research shows that primiparity [12], labor duration [13], maternal overweight [14], and perceptions about breast milk production [15] are factors associated with delays in the initiation of breastfeeding. Child birth delivery mode is another factor related to breastfeeding success. Cesarean delivery is certainly a lifesaving intervention for a mother, fetus, or both but this surgery negatively affects breastfeeding initiation and continuation [2,16-20].

Another factor that can undermine breastfeeding and the establishment of exclusive breastfeeding is prelacteal feeding [21]. In some settings, prelacteal feeds include the ceremonial provisioning of honey or rice, whereas in others it may involve water, teas, or milks [22] which can lead to early introduction with solid or semi-solid foods [23-25]. Even in the absence of prelacteal feeding, the duration of exclusive breastfeeding is often shortened by the early introduction of other liquids, solid or semi-solid foods. For example, a large survey of a rural coastal Tanzania community showed that while the mean duration of breastfeeding was two years, the average duration of exclusive breastfeeding was only 23 days as most infants were introduced to semi-solids in the form of grain-based porridges within the first two months [26]. Highlighting the importance of the first month of life, a study conducted in Peru reported that patterns in the first month of life predicted patterning in feeding throughout infancy [27]. Although less explored from an epidemiological perspective there is evidence to suggest that maternal distress associated with economic and social hardships and food insecurity plays an important role in infant feeding decisions [28-32].

The data used for our analyses were collected as part of a prospective longitudinal birth cohort study called Malnutrition and Enteric Infections: Consequences for Child Health and Development [33]. The goal of the MAL-ED study is to examine inter-relationships between diet and enteric infections over time as these relate to the development of intestinal inflammation, malnutrition and cognition in children from birth to 24 months of age. The eight MAL-ED sites are epidemiologically diverse, low-income, and located in urban (Bangladesh, Brazil, India, Nepal), peri-urban (South Africa) and rural contexts (Tanzania, Pakistan, Peru). Throughout the manuscript the sites are referred to by the following abbreviations: BGD: Dhaka, Bangladesh [34]; BRF: Fortaleza, Brazil [35]; INV: Vellore, India [36]; NEB: Bhaktapur, Nepal [37]; PEL: Loreto, Iquitos, Peru [38]; PKN: Naushero Feroze, Pakistan [39]; SAV: Venda, South Africa [40]; TZH: Haydom, Tanzania [41]. In this paper, we report and compare infant feeding experiences in the first month of life for 2,053 infants participating in MAL-ED study.

\section{Methods}

To ensure standardized data collection, the MAL-ED Network developed common and harmonized protocols and methods to be deployed at all sites. Protocols were approved by Institutional Review Boards at each of the participating research sites and at collaborating institutions and written informed consent was obtained for each participant at each site.

The goal for each site was to recruit, retain, and follow 200 mother-newborn dyads for 24 months; the actual enrollment varied between sites to because of site-specific estimates for retention until 24 months of age. The MAL-ED sample size was selected to test hypotheses about the influences on child growth and development in the first two years of life at both the all site $(\mathrm{N}=$ 1600) and individual site level.

Trained study personnel identified pregnant women through a community survey as well as through health clinics or by referral. Prior to data collection, it was decided that final eligibility would be determined based on the following inclusion criteria: 1 ) healthy singleton newborn ( $\leq 17$ days of age); 2 ) mother 16 + years of age; 3 ) family intended to stay in the study area for the next 6month period; 4) no other child from the same family was enrolled in the study; 5) birth weight or enrollment weight was greater than 1500 grams; 6) child did not have a diagnosable congenital disease or severe neonatal disease. Although enrollment at all sites ended February 2012, sites varied as to the date when they started recruiting subjects with the earliest beginning in November 2009. Furthermore, enrollment was staggered across sites to allow for analysis of, and control for, seasonality. In total, 2,145 children were enrolled in the study.

At the time of enrollment, a structured questionnaire answered by the mother was used to collect recall data on breastfeeding initiation, the withholding of colostrum, prelacteal feeding, as well as infant, maternal and family factors including infant gender and birth weight, maternal age, parity, education and marital status. Following standard protocols [42] newborn/infant anthropometric measures (length, weight and head circumference) were taken by trained study personnel. Due to limitations on protocol changes, we were able to re-interview women at 7 of the 8 sites to collect information on type of delivery (vaginal, Cesarean). We did not collect information on the HIV status of the mothers or their infants.

After enrollment, each household was visited two times each week to collect data about illnesses (since the last contact, up to 7 days), and infant feeding practices based on the previous day's pattern were recorded. Caregivers were specifically queried about the infant's consumption of the following items in the previous 24 hours: breast milk, animal milk, formula, water, tea, fruit juice, other liquids, or semi-solids. On a monthly basis, a third structured 
questionnaire was administered and anthropometrics and information about infant feeding, immunizations, and delivery of micronutrient supplements were recorded. For this paper, we compiled the infant feeding data from the three structured questionnaires (enrollment, surveillance visits, month 1 visit) to produce a comprehensive depiction of infant feeding in the first month of life [43]. Across the sites, the median number of assessments of infant feeding per child during the first month of life varied from 6 to 9 across the sites.

Median age at enrollment at the eight sites ranged from 3 to 11 days. Of the 2,142 infants enrolled, 2,077 remained in the study during the first month, and 65 were lost to follow up (7 died, 19 moved, 13 dropped out and 26 were missing information after the enrollment visit). Of the 7 infants who died, 3 were from PKN and 1 each from BGD, INV, SAV and TZH; 6 died from infectious causes and 1 died from a congenital condition. To create a profile of infant feeding during the first month, we considered as the final visit for the period as any visit on days 28-33. There were 23 infants who had no visit between days 28 and 33 and were excluded from further analyses. Many infants had more than one visit between days 28 and 33; we therefore selected the visit closest to day 30 to represent the final visit to close out the first month. We excluded one additional infant who was visited fewer than three times after enrollment, leaving a total sample of 2,053 in the final analyses. Infants excluded were from all sites, but $51 \%$ of those excluded were from the SAV site. When enrollment characteristics of the 89 infants excluded from analyses were compared to those included, the only difference found was that the mothers of excluded infants were more educated, and this can be explained by the fact that the women in the SAV site are more highly educated than women in any other site.

We followed standard definitions to characterize breastfeeding status [44] at enrollment and each visit. Prelacteal feeding was defined as anything fed to the newborn before they were breastfed for the first time. Colostrum referred to the first milk that is in the breast or is produced immediately after childbirth. Exclusive breastfeeding was defined as breastfeeding without the introduction of other food or liquids (not even water) over the prior 24-hour period, with the exception of drops or syrups consisting of vitamins, mineral supplements or medicine. If the infant received plain water or water-based liquids such as tea or juice, this was considered predominant breastfeeding. Full breastfeeding referred to either exclusive or predominant breastfeeding. The inclusion of other milks, formula and/ or semi-solids was considered partial breastfeeding. We did not consider the giving of a prelacteal feeding to nullify exclusive breastfeeding once breastfeeding was initiated. Some infants were completely weaned from the breast during the first month; when this occurred near the end of the first month, we utilized the data collected beyond the first month for confirmation.

Data were double-entered at each site by trained personnel, and were uploaded to the Data Coordination Center (DCC) on a weekly basis. Consistency checks and data cleaning were accomplished at the sites and at the DCC. Additional consistency checks and data cleaning were performed at the DCC level and sent back for investigation or correction by the corresponding site.

Data analyses were performed using STATA Version 11.0 (StataCorp LP, College Station, TX). We examined the distributions of variables, and characterized their distributions by means and standard deviations, or frequency tables as appropriate. Distributions that were not normally distributed were characterized by median and inter-quartile range (IQR) or by other percentiles of the distribution as appropriate. Because one of the goals for the analysis was to characterize the infant feeding practices in the first month of life across the eight sites, we did not focus on between-site statistical comparisons. We utilized survival analysis with age in days as the unit of analysis to characterize the pattern of decline in exclusive breastfeeding during the first month of life. Exclusive breastfeeding was treated as a one-time failure event and consequently, the child did not contribute to the risk set subsequent to the first time they departed from the exclusive breastfeeding criteria. We conducted bivariate and multivariate logistic regression analyses to examine the factors associated with partial (or no) breastfeeding at one month of age. Infant, maternal and family characteristics were evaluated for their association with partial breastfeeding at one month of age within each site as well as across all sites in an overall logistic model, which was estimated with Generalized Estimation Equations (GEE) with terms for each site as random effect included in the model. Variables with $\mathrm{p}<0.10$ were retained in the final model.

\section{Results}

Selected characteristics of the study infants and their mothers at enrollment are shown in Table 1. The median age of enrollment varied from 3-12 days across the eight sites. The mean weight of infants at enrollment ranged from a low of 2,760 $\mathrm{g}$ in BGD to a high of 3,430 $\mathrm{g}$ in BRF. Most sites were able to comprehensively collect birth weight data (97\% or greater), but only $72 \%$ from PKN and $50 \%$ from TZH had recorded birth weights because fewer enrolled infants were born at health facilities. When recorded (data not shown), the incidence of low birth weight ranged from $5-10 \%$ in BRF, INV, NEB, PEL, SAV, and $\mathrm{TZH}$, with slighter higher rates, of about $20 \%$, for BGD and PKN. Although mean maternal age was similar across the study sites, parity was much more variable across sites. 
Table 1 Selected maternal and newborn characteristics ${ }^{1}$ at enrollment by MAL-ED study site $(n=2053)$

\begin{tabular}{|c|c|c|c|c|c|c|c|c|}
\hline MAL-ED study sites ${ }^{2}$ & BGD & INV & NEB & PKN & BRF & PEL & SAV & TZH \\
\hline $\mathrm{N}$ & 256 & 242 & 238 & 269 & 231 & 293 & 268 & 256 \\
\hline Male child (\%) & 49.6 & 43.8 & 53.8 & 48.3 & 51.5 & 52.9 & 48.5 & 49.6 \\
\hline Age (d) & $3(0,9)$ & $10[3,16]$ & $12[5,16]$ & $11[2,16]$ & $9[3,16]$ & $4[2,14]$ & $8[2,16]$ & $7[3,16]$ \\
\hline Weight (g) & $2,760(400)$ & $2,920(460)$ & $3,150(460)$ & $2,890(490)$ & $3,430(510)$ & $3,090(460)$ & $3,290(480)$ & $3,370(470)$ \\
\hline Maternal age (y) & $24.9(5.1)$ & $24.1(3.8)$ & $26.5(3.7)$ & $27.6(6.0)$ & $24.8(5.5)$ & $24.2(6.1)$ & $26.4(6.9)$ & $28.5(6.7)$ \\
\hline \multicolumn{9}{|l|}{ Parity (\%) } \\
\hline 1 & 40.6 & 33.7 & 44.1 & 23.0 & 32.0 & 39.3 & 39.1 & 12.5 \\
\hline $2-4$ & 57.0 & 61.3 & 54.6 & 50.2 & 58.9 & 47.4 & 53.3 & 46.5 \\
\hline$>4$ & 2.4 & 5.0 & 1.3 & 26.8 & 9.1 & 13.3 & 7.6 & 41.0 \\
\hline Cesarean delivery $(\%)^{3}$ & 21.5 & 9.8 & 18.9 & 14.5 & 55.7 & 8.9 & 8.8 & - \\
\hline \multicolumn{9}{|l|}{ Maternal education (y) } \\
\hline $0-5$ & 64.1 & 37.6 & 25.6 & 81.8 & 8.2 & 21.8 & 1.5 & 37.5 \\
\hline $6-10$ & 34.8 & 47.1 & 52.1 & 12.3 & 36.8 & 57.7 & 47.8 & 62.5 \\
\hline $11-15$ & 1.2 & 15.3 & 22.3 & 5.9 & 55.0 & 21.5 & 50.7 & 0.0 \\
\hline
\end{tabular}

${ }^{1}$ Presented are mean (SD) or \%, except for age at enrollment for which median $\left(5^{\text {th }}, 95^{\text {th }}\right.$ percentiles) are shown.

${ }^{2}$ Abbreviations: BGD Dhaka, Bangladesh; BRF Fortaleza, Brazil; INV Vellore, India; NEB Bhaktapur, Nepal; PKN Naushero Feroze, Pakistan; PEL Loreto, Peru; SAV Venda, South Africa; TZH Haydom, Tanzania.

${ }^{3}$ Data not available for 18 participants in INV, 39 in BRF and 63 in SAV. No data available for TZH.

In TZH and PKN, $41 \%$ and $27 \%$ of mothers, respectively, were grand multipara, and only $13 \%$ and $23 \%$, respectively, were primipara. In contrast, at the other sites, $32-44 \%$ of study mothers were primipara. Education varied across sites; the proportion of mothers with low education, or less than 5 years of schooling, ranged from a high of $82 \%$ in PKN to a low of only $2 \%$ in SAV.

Characteristics of the early breastfeeding practices are shown in Table 2 . At the PKN site, only $7.4 \%$ were put to the breast in the first hour, and $20.9 \%$ did not initiate until day 1 or later. At the other seven sites, rates were higher with $41-83 \%$ of infants being put to the breast within one hour of birth, and $90-98 \%$ breastfed within 24 hours of birth. In SAV, 6 (2.2\%) infants never received breast milk. In BGD, BRF, NEB, PEL and SAV, less than $5 \%$ of mothers reported not giving their infant colostrum, but in TZH, PKN and INV the reported rates were higher, ranging from 8.6 to $16.4 \%$. In $\mathrm{PKN}, 63.2 \%$ of

Table 2 Selected characteristics of early breastfeeding practices by MAL-ED study site

\begin{tabular}{|c|c|c|c|c|c|c|c|c|}
\hline MAL-ED study sites ${ }^{1}$ & $\mathrm{BGD}$ & INV & NEB & $\mathrm{PKN}$ & BRF & PEL & SAV & $\mathrm{TZH}$ \\
\hline \multicolumn{9}{|l|}{ Initiation (\%) } \\
\hline Within 1 hour & 60.9 & 59.1 & 40.8 & 7.4 & 46.3 & 73.7 & 59.7 & 83.2 \\
\hline $1-24 h$ & 33.6 & 36.4 & 49.2 & 71.8 & 51.5 & 23.9 & 35.5 & 14.8 \\
\hline $1-3 d$ & 5.1 & 4.5 & 9.2 & 18.6 & 1.3 & 1.4 & 2.6 & 1.6 \\
\hline $4+d$ & 0.4 & 0.0 & 0.8 & 2.3 & 0.9 & 1.0 & 0.0 & 0.4 \\
\hline Never & 0.0 & 0.0 & 0.0 & 0.0 & 0.0 & 0.0 & 2.2 & 0.0 \\
\hline Not fed colostrum (\%) & 1.6 & 9.9 & 3.4 & 16.4 & 1.7 & 2.7 & 4.5 & 8.6 \\
\hline Prelacteal feeding (\%) & 13.3 & 12.4 & 17.7 & 63.2 & 6.9 & 9.2 & 3.7 & 5.1 \\
\hline Exclusively Breastfed at $30 \mathrm{~d}(\%)^{2}$ & 84.7 & 81.0 & 55.5 & 4.5 & 59.7 & 38.2 & 29.5 & 55.9 \\
\hline Fully Breastfed at $30 \mathrm{~d}(\%)^{2,3}$ & 90.6 & 87.6 & 70.6 & 7.4 & 68.4 & 77.1 & 58.6 & 58.2 \\
\hline Partially Breastfed at $30 \mathrm{~d}(\%)^{4}$ & 9.4 & 11.2 & 29.4 & 92.6 & 29.4 & 22.5 & 36.6 & 41.8 \\
\hline Completely weaned by $30 \mathrm{~d}(\%)^{5}$ & 0.0 & 1.2 & 0.0 & 0.0 & 2.2 & 0.3 & 2.6 & 0.0 \\
\hline
\end{tabular}

${ }^{1}$ Abbreviations: BGD Dhaka, Bangladesh; BRF Fortaleza, Brazil; INV Vellore, India; NEB Bhaktapur Nepal; PKN Naushero Feroze, Pakistan; PEL Loreto, Peru; SAV Venda, South Africa; TZH Haydom Tanzania.

${ }^{2}$ Based on multiple assessments through the first month of life, assessed at $\sim 30 \mathrm{~d}$.

${ }^{3}$ Those exclusively or predominantly breastfed, assessed at $\sim 30 \mathrm{~d}$.

${ }^{4}$ Those who have received formula, animal milks or semi-solids in addition to breast milk, assessed at $\sim 30 \mathrm{~d}$.

${ }^{5}$ Those no longer receiving breast milk (verified by subsequent reporting after $\sim 30 \mathrm{~d}$ ). 
infants were given a prelacteal feeding, substantially higher than the incidence of 12.4 to $17.7 \%$ in the other South Asian sites, and 3.7-9.2\% in the remaining four MAL-ED sites.

As shown, the incidence of cesarean delivery ranged from 8.8 to $55.7 \%$. Because of their known interrelationships, we examined the bi-variate associations amongst type of delivery, delay in initiation, prelacteal feeding and avoidance of colostrum. Cesarean delivery was significantly associated with the provision of a prelacteal feed at 5 of the 7 sites, and with the avoidance of colostrum at 2 of the 7 sites that collected type of delivery data. Cesarean delivery was also significantly associated with a delay ( $>1$ hour after birth) in breastfeeding initiation at 4 of 7 sites, and was suggestive $(p=0.09)$ at 1 site.

By one month of age, the proportion of infants exclusively breastfed had declined in all sites (Figure 1 and Table 2). Only in BGD and INV were the proportions greater than $80 \%$, with the remaining sites reporting proportions from a high of $59.7 \%$ (BRF) to a low $4.5 \%$ (PKN). As shown in Figure 1, the rate of decline in exclusive breastfeeding in PKN was steepest during the first 15 days, whereas in the other sites, the rate of decline was relatively stable across the time period although rates varied across sites. In PEL and SAV, the decline in exclusive breastfeeding was strongly related to the provisioning of water and water-based preparations to the infant; as shown in Table 2, the proportions of infants fully breastfed at day 30 was almost double the proportion of those exclusively breastfed in those sites (77.1\% versus $38.2 \%$ in PEL; $58.6 \%$ versus $29.5 \%$ in SAV). The results in Table 2 also indicate that other than in INV and BGD, about one-quarter or more of the infants were partially breastfed by the end of the first month, meaning that they had already received semi-solids, formula or animal milks. Some infants were completely weaned during the first month in PEL (0.3\%), INV (1.26\%), BRF (2.2\%) and SAV (2.6\%).

The proportions of infants fed plain water, different types of liquids or semi-solid foods during the first one month of life are presented in Figure 2. Formula was reportedly given to some infants in seven of the eight sites, with the highest proportions reported in SAV (20\%), BRF (19\%) and NEB (15\%). Animal milks were also given to some infants in all sites, except for the SAV site where this is not part of infant feeding practices. Coffee was given to infants in PEL and BRF, and tea was given to infants in PEL, PKN and INV. Teas prepared in PKN and INV usually included animal milks whereas teas prepared in PEL were mostly herbal varieties without the addition of animal milks. Semi-solid porridges were given to infants in TZH, SAV and to a lesser extent in PKN. Some sites reported a high proportion of infants receiving other liquids; the identity of the other liquids varied by site and consisted of gripe or sugar water in a few sites and animal milk-based preparations (other than tea) in PKN. Additional analyses of the frequency with

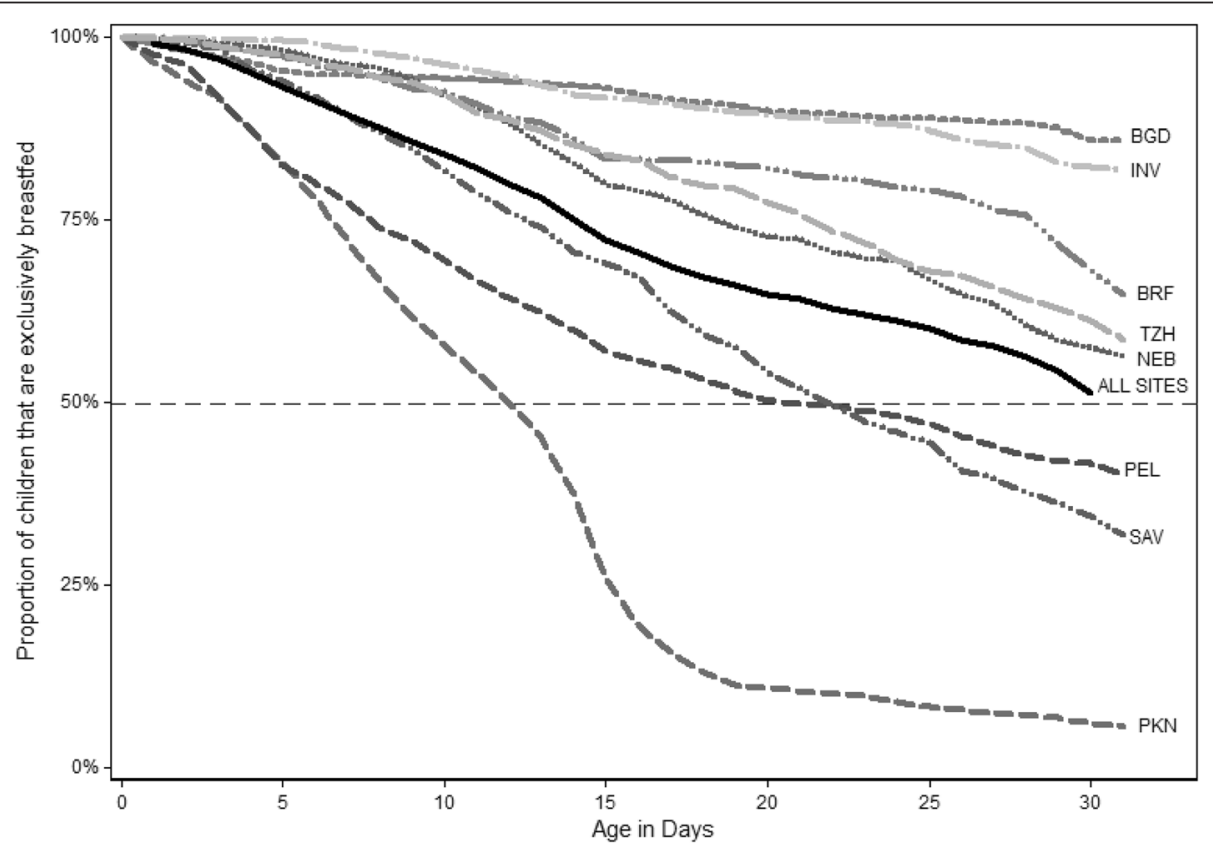

Figure 1 Decline in percent of newborns exclusively breastfed over time during the first month of life by MAL-ED site estimated through survival analysis with smoothing. Abbreviations: BGD: Dhaka, Bangladesh; BRF: Fortaleza, Brazil; INV: Vellore, India; NEB: Bhaktapur, Nepal; PKN: Naushero Feroze, Pakistan; PEL: Loreto, Peru; SAV: Venda, South Africa; TZH: Haydom, Tanzania. 


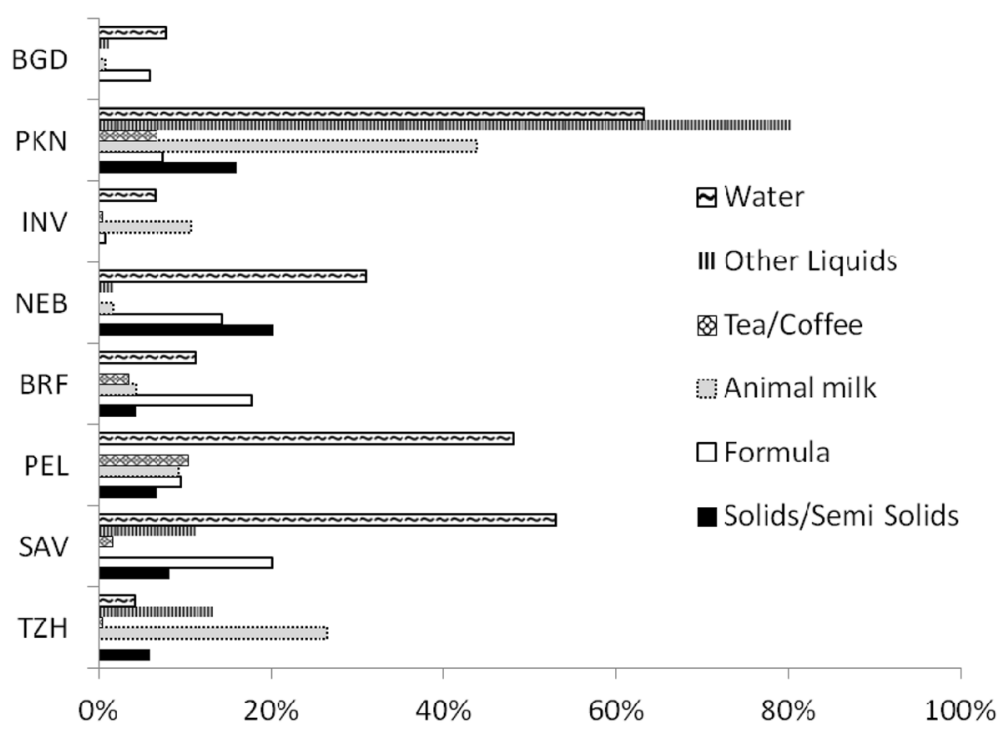

Figure 2 Semi-solids and liquids ever fed during the first month of life by MAL-ED site.Abbreviations: BGD: Dhaka, Bangladesh; BRF: Fortaleza, Brazil; INV: Vellore, India, NEB:, Bhaktapur, Nepal; PKN: Naushero Feroze, Pakistan; PEL: Loreto, Peru; SAV: Venda, South Africa; TZH: Haydom, Tanzania.

which these liquids or foods were fed (among those reported receiving them) indicated that except for BGD, these food items were incorporated into the feeding pattern for the child. For example, when plain water was given, the median frequency of provision in subsequent visits was $33-80 \%$ of visits during the first month in INV, NEB, PKN, PEL and SAV. The median frequency of reporting of formula (when initiated) was $43-87 \%$ across 5 sites, and for other animal milks the median frequency was 14-63\% across 7 sites. Among those receiving semisolids, the median frequency of consumption thereafter was $14-63 \%$ of visits.

The likelihood of partial (or no) breastfeeding at one month was associated with early feeding practices (Table 3). The provisioning of a prelacteal feed increased the risk of partial breastfeeding (Odds Ratio (OR): 1.48 (95\% Confidence Interval (CI): 1.04, 2.10), as did the withholding of colostrum (1.63: 1.01, 2.62). There was a tendency for the initiation of breastfeeding within the first hour to be associated $(\mathrm{p}=0.09)$ with decreased risk of partial breastfeeding at one month $(0.81: 0.64,1.03)$. Factors such as having the husband as head of household,

Table 3 Risk factors ${ }^{1}$ for partial (or no) breastfeeding at one month of life in the MAL-ED study

\begin{tabular}{ll}
\hline Factor & OR $(95 \% \mathrm{CI})$ \\
\hline Prelacteal feeding & $1.48(1.04,2.10)$ \\
Not fed colostrum & $1.63(1.01,2.62)$ \\
Primipara & $1.38(1.10,1.75)$ \\
Put to the breast within $1 \mathrm{~h}$ & $0.81(0.64-1.03)$ \\
\hline
\end{tabular}

${ }^{1}$ Estimated with generalized estimation equations (GEE) with sites included as random effects. This is the reduced model including variables with $p<0.10$, see text for details. maternal age and education were not associated with risk of partial breastfeeding, but being a first-time mother was indicative of higher risk of partial breastfeeding (1.38: 1.10, 1.75). Infant gender did not affect the likelihood of partial breastfeeding at one month. Being born of low birth weight $(<2500 \mathrm{~g})$ did not significantly affect risk, when evaluated in the sub-sample of infants with known birth weights $(n=1519)$. Type of delivery was also not significantly associated with risk of partial breastfeeding after adjusting for pre-lacteal feeding, withholding of colostrum, etc, in a model with data from 7 sites.

\section{Discussion}

The WHO recommends that all infants receive breast milk beginning within an hour of their birth, that they not be given a prelacteal feeding, and that they be fed only breast milk until they reach 6 months of age. As shown here, through multiple practices including delayed initiation, the withholding of colostrum, the offering of prelacteal feeding and the provision of liquids and semi-solids, the practice of optimal breastfeeding is compromised in each of these sites. There is variability in the degree and manner through which this occurs across the sites. Overall, $40-83 \%$ of study newborns were put to the breast within an hour after birth, except in the PKN site where mothers reported that this rarely occurred. Over $60 \%$ of infants received a prelacteal feeding in PKN in contrast to less than $20 \%$ elsewhere - including the three other sites in South Asia. Most - but not all newborns - were given colostrum. The proportion of infants exclusively breastfed at one month varied greatly among sites, from a low of $5 \%$ to a high of $85 \%$. In some sites, the primary reason for this decline was the provisioning 
of water or water-based preparations, however, the proportions of infants partially breastfed at one month (most commonly between $22-42 \%$ ), indicate that significant numbers of infants are receiving animal milks, alone or in preparations, and semi-solid foods. Some are already weaned from the breast. Taken together, these data provide a compelling picture of vulnerability resulting from very early care and infant feeding decisions made for these neonates living in diverse settings.

Our analyses indicate that very early infant feeding practices, which include the provisioning of prelacteal feeds and the withholding of colostrum, affect subsequent breastfeeding patterns, specifically in this case, whether or not the infant will be given other milks or semi-solids by the end of the first month of life. Both practices are known to delay the initiation of breastfeeding, and we confirm that across these sites, they increase the likelihood of partial (or no) breastfeeding in the first month. Delayed initiation ( $>1$ hour of birth) may be due to other factors as well, but as shown here was only marginally associated with increased risk of partial breastfeeding. Primipara, who are attempting breastfeeding for the first time, were also found to be at risk of moving to partial (or no) breastfeeding.

Less than optimal early infant feeding practices have implications for infant health and survival. Although many view the provision of water or teas to infants to be innocuous and not interfere with breast milk production, predominant breastfeeding has been shown to elevate risk of mortality in the first 6 months of life, and risks associated with partial breastfeeding are even higher $[45,46]$. Late initiation of breastfeeding $(>24 \mathrm{~h})$ has been shown in more than one study to increase risk of early cessation of exclusive breastfeeding and the risk of dying $[9,15]$. Prelacteal feeding exerts multiple negative consequences as well - increasing risk of cessation of exclusive breastfeeding [21], of diarrheal and respiratory illness, and of mortality $[8,11,47]$.

The MAL-ED study sites were chosen, in part, because of on-going research on enteric infections at these sites. Thus, our site data should not be interpreted as representative of the situation in the entire country or even for a broader region; however, it is worthwhile to compare our results with previously published data from these countries. Overall, the proportions of MAL-ED infants receiving prelacteal feeds were much lower than those reported nationally or for the same region within each country [24,48-54]; clear exceptions to this were PKN and NEB. In PKN, the study frequency of prelacteal feeding of $63 \%$ is in line with the regional and national incidences of 55 and $65 \%$, respectively [48], and in NEB, the incidence of $18 \%$ is similar to estimates of $17 \%$ from a recent study in the region [55] and $13.8 \%$ for another region of Nepal [56]. Incidences of early initiation of breastfeeding (within 1 hour of birth) in NEB, BRF SAV, TZH and INV were of comparable magnitude to regional (sub-national) or national figures [49,51,52,54,57], but were greater than regional/national figures in PEL and BGD [50,53], and lower than estimates in PKN [48]. In general, our sample estimates correspond to the statistics on early initiation of breastfeeding reported by WHO region [58].

It is difficult to compare the prevalence of exclusive breastfeeding at one month collected prospectively through multiple visits with national or regional survey based on a single interview and aggregated for infants $<$ 2 months of age. However, in general, the prevalence of exclusive breastfeeding at one month in the MAL-ED sites are lower than those reported for the corresponding larger region or area for five of our sites (PKN, NEB, $\mathrm{TZH}, \mathrm{SAV}, \mathrm{PEL})[48-51,54]$, but higher in the three other sites (INV, BRF, BGD) [52,53,57].

There are numerous strengths to the present study design and methodology. We used a common, harmonized protocol to collect comparable information in eight unique study sites all located in developing regions of the world. Study personnel were trained and various consistency checks and control measures enhanced data quality. Mothers were queried just after delivery and twice weekly regarding their infant feeding practices, allowing us to collect current information as feeding practices changed with time.

The main limitation of this study is that it was not designed specifically to assess risk factors associated with partial breastfeeding. This paper results from a post hoc analyses of longitudinal data collected as part of the larger MAL-ED study - a study designed to examine the influences of enteric infections (diarrhea), dietary intake, and gut function on infant growth and development. For the analyses presented here, we examined the minimal detectable differences in the odds for various risk factors. For all-site analyses, with $80 \%$ power and an alpha level of 0.05 , we are able to detect differences in the odds greater than $20 \%$, and for individual site analyses, differences of $80 \%-200 \%$ are detectable depending on the site and risk factor. A study designed to examine risk factors for partial breastfeeding would likely have included additional factors that were not collected as part of the MAL-ED study. However, given the variation in breastfeeding practices, we were able to identify multiple salient factors influencing partial breastfeeding in the first month across the 8 sites.

Another component of this limitation is that infants were enrolled from birth to 17 days, and thus, the period of maternal recall of initiation and the level of certainty regarding breastfeeding practices in the early days varies across the sites. However, $55 \%$ of infants were enrolled by 7 days of life, and it should be noted that the longer 
period of time from birth to enrollment likely makes our results paint a more optimistic picture of breastfeeding practices in these sites, because we might have missed the earliest interruptions in exclusive breastfeeding. Also, missing information on birth weight and type of delivery at some sites precluded our ability to fully investigate these risk factors. Last, we did not collect additional information at each visit that would allow us to understand the reasons for the reported feeding practices. The MAL-ED study was designed to be an observational study, and we did not want to call attention to specific practices and thereby influence the results. Ultimately, we wish to detect differences in early infant feeding practices in order to relate this information to other data on gut function, enteric illness, child growth and development, all of which are being simultaneously collected in the study.

Across these eight MAL-ED sites, breastfeeding is nearly universal, and the generally rapid initiation of breastfeeding, the low frequency with which prelacteal feeding or the withholding of colostrum are reported, all support optimal feeding and infant health. Yet, liquids were introduced frequently to infants in the first month of life and in some sites semi-solids were beginning to appear in the young infant's diet. We show that when breastfeeding initiation is not the ideal, and when the mother is inexperienced, there is increased risk for an early move away from exclusive breastfeeding. Infant feeding practices develop as a result of multiple influences attributable to the mother-child dyad, the extended family, the broader local culture as well as from public health or health care provider messages which seek to promote health [59]. In some sites, such as in Peru, Brazil, India and Bangladesh, a relatively well developed care setting exists, whereas for sites in Nepal, South Africa, Tanzania and Pakistan little to no programmatic activity exists to promote optimal breastfeeding practices. The Baby-Friendly Hospital Initiative (BFHI) was initiated in 1990s to strengthen health facility support for the establishment of early and exclusive breastfeeding through adherence to 10 principles for optimal breastfeeding [60]. Early support/teaching to establish good technique particularly for the first-time mother, no prelacteal feeding, the giving of colostrum, putting the baby to the breast within 30 minutes of delivery are important principles in that strategy. Some have expanded the framework to the community $[61,62]$, and in $2009 \mathrm{WHO} / \mathrm{UNICEF}$ revised and expanded this initiative [63]. Our results underscore the need to implement and/or strengthen these and other proven programmatic efforts in both maternity and pediatric services to support and protect exclusive breastfeeding between birth and the likely first postnatal pediatric care visit. The short and long-term implications of the early feeding experiences reported here on enteric illnesses, and child growth and development will be the subject of additional analyses of MAL-ED data.

\section{Additional file}

Additional file 1: MAL-ED Network Investigators and Institutional Affiliations.

\section{Competing interests}

The authors declare that they have no competing interest.

\section{Authors' contributions}

CLP oversaw data collection in the Tanzania site, participated in the interpretation of results, and drafting the manuscript. AT oversaw data collection in the Pakistan site, participated in the interpretation of results, and drafting the manuscript. RA conducted the statistical analysis and participated in drafting the manuscript. CN, oversaw data collection in the South Africa site, and participated in the interpretation of results. RKC oversaw data collection in the Nepal site, and participated in the interpretation of results. AB oversaw data collection in the India site, and participated in the interpretation of results. MMI and AMSA oversaw data collection in the Bangladesh site, and participated in the interpretation of results. MPO oversaw data collection in the Peru site and participated in the interpretation of results. MLM oversaw data collection in the Brazil site, and participated in the interpretation of results. LEC provided technical assistance in data collection to all sites, participated in the analysis and interpretation of results and drafting the manuscript. All authors read and approved the final manuscript.

\section{Acknowledgements}

The Etiology, Risk Factors and Interactions of Enteric Infections and Malnutrition and the Consequences for Child Health and Development Project (MAL-ED) is carried out as a collaborative project supported by the Bill \& Melinda Gates Foundation, the Foundation for the $\mathrm{NIH}$ and the National Institutes of Health/ Fogarty International Center. The authors thank the staff and participants of the MAL-ED Network Project for their important contributions.

\section{Author details}

${ }^{1}$ Department of Women, Children and Family Health Science, College of Nursing, University of Illinois at Chicago, Chicago, IL, USA. ${ }^{2}$ Department of Pediatrics and Child Health, Aga Khan University, Karachi, Pakistan. ${ }^{3}$ Fogarty International Center, National Institutes of Health, Bethesda, MD, USA.

${ }^{4}$ Department of Nutrition, School of Health Sciences, University of Venda, Thohoyandou, Limpopo Province, South Africa. ${ }^{5}$ Centre for International Health, University of Bergen, Norway and Department of Child Health and Institute of Medicine, Tribhuvan University, Kathmandu, Nepal. ${ }^{6}$ Christian Medical College, Vellore, India. ${ }^{7}$ Centre for Nutrition and Food Security, International Centre for Diarrhoeal Disease Research, Dhaka, Bangladesh. ${ }^{8}$ Biomedical Investigations Unit AB PRISMA, Iquitos, Peru. ${ }^{9}$ Department of Nutrition, State University of Ceará, Fortaleza, Ceará, Brazil. ${ }^{10}$ The Johns Hopkins Bloomberg School of Public Health, Department of International Health, 615 North Wolfe Street, W2041, Baltimore, MD, USA21205.

Received: 11 December 2013 Accepted: 24 February 2015 Published online: 01 May 2015

\section{References}

1. Jones G, Steketee R, Black R, Bhutta ZA, Morris SS, Group BCSS. How many child deaths can we prevent this year? Lancet. 2003;362:65-71.

2. Kramer MS, Chalmers B, Hodnett ED. Promotion of breastfeeding intervention trial (probit): A randomized trial in the republic of belarus. JAMA J Am Med Assoc. 2001;285(4):413-20.

3. WHO. Effect of breastfeeding on infant and child mortality due to infectious diseases in less developed countries: a pooled analysis. WHO Collaborative Study Team on the Role of Breastfeeding on the Prevention of Infant Mortality. Lancet. 2000;55(9202):451-5.

4. Victora C, Vaughan JP, Lombardi C, Fuchs SC, Gigante L, Smith P, et al. Evidence for protection by breast-feeding against infant deaths from infectious diseases in Brazil. Lancet. 1987;330(8554):319-22. 
5. Kramer MS, Kakuma R. The optimal duration of exclusive breastfeeding. In: Pickering LK, Morrow AL, Ruiz-Palacios G, Schanler RJ, editors. Protecting Infants through Human Milk: Advancing Scientific Evidence. New York: Kluwer; 2004.

6. Black RE, Allen LH, Bhutta ZA, Caulfield LE, de Onis M, Ezzati M, et al. Maternal and child undernutrition: global and regional exposures and health consequences. Lancet. 2008;371(9608):243-60.

7. Caulfield LE, de Onis M, Blassner M, Black RE. Undernutrition as an underlying cause of child deaths associated with diarrhea, pneumonia, malaria, and measles. Am J Clin Nutr. 2004;80(1):193-8.

8. Edmond KM, Kirkwood BR, Tawiah CA, Agyei SO. Impact of early infant feeding practices on mortality in low birth weight infants from rural Ghana. J Perinatol. 2008;28(6):438-44.

9. Garcia CR, Mullany LC, Rahmathullah L, Katz J, Thulasiraj RD, Sheeladevi S, et al. Breast-feeding initiation time and neonatal mortality risk among newborns in South India. J Perinatol. 2011;31(6):397-403.

10. Mullany LC, Katz J, Li YM, Khatry SK, LeClerq SC, Darmstadt GL, et al. Breast-feeding patterns, time to initiation, and mortality risk among newborns in southern Nepal. J Nutr. 2008;138(3):599-603.

11. Savitha MR, SB N, PK MJ, ul-Haque F, Raju CK. Modifiable risk factors for acute lower respiratory tract infections Indian. J Pediatr. 2007;74(5):477-82.

12. Chapman DJ, Perez-Escamilla R. Identification of risk factors for delayed onset of lactation. J Am Diet Assoc. 1999;99(4):450-4.

13. Chen DC, Nommsen-Rivers L, Dewey KG, Lönnerdal B. Stress during labor and delivery and early lactation performance. Am J Clin Nutr. 1998;68(2):335-44.

14. Rasmussen KM, Hilson JA, Kjolhede CL. Obesity May impair lactogenesis II. J Nutr. 2001;131(11):3009S-3011.

15. Tawiah-Agyemang C, Kirkwood BR, Edmond K, Bazzano A, Hill Z. Early initiation of breast-feeding in Ghana: barriers and facilitators. J Perinatol. 2008;28(S2):S46-52.

16. WHO/UNICEF. WHO | Protecting, promoting and supporting breast-feeding Geneva: World Health Organization; 1989.

17. Patel RR, Liebling RE, Murphy DJ. Effect of operative delivery in the second stage of labor on breastfeeding success. Birth Blackwell Science Inc; 2003;30(4):255-60

18. Perez-Escamilla R, Moaulen-Radovan I, Dewey KG. The association between cesarean delivery and breast-feeding outcomes among Mexican women. Am J Public Health. 1996:86(8):832-6.

19. Vieira T, Vieira G, Giugliani E, Mendes C, Martins C, Silva L. Determinants of breastfeeding initiation within the first hour of life in a Brazilian population: cross-sectional study. BMC Public Health. 2010;10(1):760.

20. Vestermark $V$, Hogdall CK, Birch M. al. et. Influence of the mode of delivery on initiation of breast-feeding. Eur J Obstet Gynecol Reprod Biol. 1991;38(1):33-8.

21. Perez-Escamilla R, Segura-Millan S, Canahuati J, Allen H. Prelacteal feeds are negatively associated with breast-feeding outcomes in Honduras. J Nutr. 1996;126(11):2765-73.

22. Greiner $\mathrm{T}$, Latham MC. Infant feeding practices in St. Vincent and factors which affect them West Indian Med J. 1981;30(1):8-16.

23. Ludvigsson J. Breastfeeding intentions, patterns, and determinants in infants visiting hospitals in La Paz. Bolivia BMC Pediatr. 2003;3(1):5.

24. Marques NM, Lira PIC, Lima MC, da Silva NL, Filho MB, Huttly SRA, et al. Breastfeeding and early weaning practices in northeast Brazil: a longitudinal study. Pediatrics. 2001;108(4), e66.

25. WHO. Complementary feeding of young children in developing countries: A review of current scientific knowledge. Geneva, Switzerland: WHO; 1998.

26. Shirima R, Greiner T, Kylberg E, Gebre-Medhin M. Exclusive breast-feeding is rarely practised in rural and urban Morogoro, Tanzania. Public Health Nutr. 2001:4(2):147-54.

27. Piwoz EG, Black RE, de Romaña GL, de Kanashiro HC, Brown KH. The relationship between infants' preceding appetite, illness, and growth performance and mothers' subsequent feeding practice decisions. Soc Sci Med. 1994;39(6):851-60.

28. Webb-Girard A, Cherobon A, Mbugua S, Kamau-Mbuthia E, Amin A, Sellen DW. Food insecurity is associated with attitudes towards exclusive breastfeeding among women in urban Kenya. Matern Child Nutr Blackwell Publishing Ltd; 2012;8(2):199-214.

29. Farmer P. bad blood, spoiled milk: bodily fluids as moral barometers in rural Haiti. Am Ethnol Blackwell Publishing Ltd. 1988;15(1):62-83.

30. Tapias M. Emotions and the Intergenerational Embodiment of Social Suffering in Rural Bolivia. Med Anthropol Q Blackwell Publishing Ltd; 2006;20(3):399-415.
31. Maternowska MC. Reproducing inequities: poverty and the politics of population in Haiti. New Brunswick: Rutgers University Press; 2006.

32. Gray SJ. Ecology of weaning among nomadic Turkana pastoralists of Kenya - maternal thinking, maternal behavior, and human adaptive strategies. Hum Biol. 1996;68(3):437-65.

33. The MAL-ED. Network. The MAL-ED study: a multinational and multidisciplinary approach to understand the relationship between enteric pathogens, malnutrition, Gut physiology, physical growth, cognitive development, and immune responses in infants and children Up to 2 years of. Clin Infect Dis. 2014;59 suppl 4:S193-206.

34. Ahmed T, Mahfuz M, Islam MM, Mondal D, Hossain Ml, Ahmed AMS, et al. The MAL-ED cohort study in mirpur. Bangladesh Clin Infect Dis. 2014;59 suppl 4:S280-6.

35. Lima AAM, Oriá RB, Soares AM, Filho JQ, de Sousa F, Abreu CB, et al. Geography, population, demography, socioeconomic, anthropometry, and environmental status in the MAL-ED cohort and case-control study sites in Fortaleza, ceará. Brazil Clin Infect Dis. 2014;59 suppl 4:S287-94.

36. John SM, Thomas RJ, Kaki S, Sharma SL, Ramanujam K, Raghava MV, et al. Establishment of the MAL-ED birth cohort study site in Vellore. Southern India Clin Infect Dis. 2014;59 suppl 4:S295-9.

37. Shrestha PS, Shrestha SK, Bodhidatta L, Strand T, Shrestha B, Shrestha R, et al. Bhaktapur, Nepal: the MAL-ED birth cohort study in Nepal. Clin Infect Dis. 2014:59 suppl 4:S300-3.

38. Yori PP, Lee G, Olórtegui MP, Chávez CB, Flores JT, Vasquez AO, et al. Santa Clara de nanay: the MAL-ED cohort in Peru. Clin Infect Dis. 2014;59 suppl 4:S310-6.

39. Turab A, Soofi SB, Ahmed I, Bhatti Z, Zaidi AKM, Bhutta ZA. Demographic socioeconomic, and health characteristics of the MAL-ED network study site in rural Pakistan. Clin Infect Dis. 2014;59 suppl 4:S304-9.

40. Bessong PO, Nyathi E, Mahopo TC, Netshandama V. Development of the dzimauli community in Vhembe district, Limpopo province of south africa, for the MAL-ED cohort study. Clin Infect Dis. 2014;59 suppl 4:S317-24.

41. Mduma ER, Gratz J, Patil C, Matson K, Dakay M, Liu S, et al. The etiology, risk factors, and interactions of enteric infections and malnutrition and the consequences for child health and development study (MAL-ED): description of the Tanzanian site. Clin Infect Dis. 2014;59 suppl 4:S325-30.

42. Lohman TG, Roche AF, Martorell R. Anthropometric standardization reference manual. Human Kinetics: Champaigb, IL; 1998.

43. Caulfield LE, Bose A, Chandyo RK, Nesamvuni C, de Moraes ML, Turab A, et al. Infant feeding practices, dietary adequacy, and micronutrient status measures in the MAL-ED study. Clin Infect Dis. 2014;59 suppl 4:S248-54.

44. Labbok M, Krasovek K. Toward consistency in breastfeeding definitions. Stud Fam Plann. 1990;221(4):226-30.

45. Black RE, Morris SS, Bryce J. Where and why are 10 million children dying every year? Lancet. 2003;361(9376):2226-34.

46. Doak CM, van der Starre RE, van Beusekom I, Campos Ponce M, Vossenaar $\mathrm{M}$, Solomons NW. Earlier introduction of aguitas is associated with higher risk of stunting in infants and toddlers in the Western Highlands of Guatemala. Am J Clin Nutr. 2013;97(3):631-6

47. Huffman SL, Combest C. Role of breast-feeding in the prevention and treatment of diarrhoea. J Diarrhoeal Dis Res. 1990;8(3):68-81.

48. National Institute of Population Studies (NIPS) [Pakistan] and MII. Pakistan Demographic and Health Survey 2006-07. 2008.

49. Tanzania, National Bureau of Statistics (NBS) and ORC Macro. Tanzania Demographic and Health Survey, 2009. 2010.

50. Instituto Nacional de Estadística e Informática Peru. Encuesta Demográfica y de Salud Familiar (ENDES) Continua 2004-2006. 2006

51. Nepal Ministry of Health and Population, New ERA and MII. Nepal Demographic and Health Survey 2010. 2011.

52. International Institute for Population Sciences (IIPS) and Macro International Inc. India Demographic and Health Survey 2007. 2008.

53. National Institute of Population Research and Training (NIPORT), Mitra and Associates, ORC Macro: Bangladesh, Calverton M. Bangladesh Demographic and Health Survey 2007. 2008.

54. DOH. South Africa Demographic and Health Survey. Macrointernational Inc. 2003;

55. Ulak M, Chandyo R, Mellander L, Shrestha P, Strand T. Infant feeding practices in Bhaktapur, Nepal: a cross-sectional, health facility based survey. Int Breastfeed J. 2012;7(1):1

56. Chandrashekhara TS, Joshia HS, Binua VS, Shankara PR, Ranaa MS, Ramachandrana U. Breast-feeding initiation and determinants of exclusive breast-feeding - a questionnaire survey in an urban population of western Nepal. Public Health Nutr. 2007;10(2):192-7. 
57. UNICEF. State of the World's Children. New York: UNICEF; 2009.

58. Lutter CK, Daelmans BMEG, de Onis M, Kothari MT, Ruel MT, Arimond M, et al. Undernutrition, poor feeding practices, and Low coverage of Key nutrition interventions. Pediatr. 2011;128(6):e1418-27.

59. Venancio SI, Saldiva SRDM, Escuder MML, Giugliani ERJ. The baby-friendly hospital initiative shows positive effects on breastfeeding indicators in brazil. J Epidemiol Community Health. 2012;66(10):914-8.

60. WHO/UNICEF. Protecting, Promoting and Supporting Breastfeeding: The special Role of Maternity Services. A joint WHO/UNICEF Statement. Geneva, Switzerland: World Health Organization; 1989.

61. Casanovas C, Saadeh R. Scaling up protection, promotion, and support of breastfeeding at the community level. Food Nutr Bull. 2009:30(2 Suppl):S230-5

62. Radford A, Rickitt C, Williams A. Breast feeding: the baby friendly initiative. Unicef's baby friendly initiative is making great progress in UK. BMJ. 1998;317(7169):1385.

63. WHO/UNICEF. WHO | Baby-Friendly Hospital Initiative Revised, updated and expanded for integrated care. Geneva, Switzerland: World Health Organization; 2009.

\section{Submit your next manuscript to BioMed Central and take full advantage of:}

- Convenient online submission

- Thorough peer review

- No space constraints or color figure charges

- Immediate publication on acceptance

- Inclusion in PubMed, CAS, Scopus and Google Scholar

- Research which is freely available for redistribution 